\title{
Autoaumento vesical por laparoscopia. Modelo experimental en conejos (oryctolagus cuniculus)
}

\author{
Sánchez-Salas RE*, Palmer-Román KJ*, Dávila Barrios H*, Sánchez-Ismayel A**, Miquilarena R***. \\ *Servicio de Urología. Hospital Universitario de Caracas, UCV. **Servicio de Cirugía III, \\ ***Instituto de Cirugía Experimental, UCV.
}

Actas Urol Esp. 2008;32(7):722-726

\section{RESUMEN}

AUTOAUMENTO VESICAL POR LAPAROSCOPIA. MODELO EXPERIMENTAL EN CONEJOS (ORYCTOLAGUS CUNICULUS)

Introducción: La vejiga pequeña y contraída representa un cuadro clínico de difícil terapéutica y no siempre efectiva. Se presenta la cirugía preservadora de urotelio como una opción que permite el aumento de la capacidad vesical. Nuestro objetivo fue evaluar la factibilidad del conejo (Oryctolagus cuniculus) como modelo experimental de enseñanza, entrenamiento y aplicación de técnica para el autoaumento vesical por laparoscopia.

Material y Métodos: Cinco conejos (Oryctolagus cuniculus), machos, New Zealand, fueron intervenidos laparoscópicamente por técnica transperitoneal de tres puertos, realizándose autoaumento vesical mediante detrusorectomía. Se realizó cistometría simple preoperatoria y postoperatoria inmediata, mediata y tardía, para verificar modificaciones de capacidad vesical.

Resultados: 5 conejos machos fueron operados en un tiempo promedio de 68,8 minutos (rango 44-120). Se realizó medición de la capacidad vesical preoperatoria con promedio de 25.8cc (7-52) disminuyendo la postoperatoria inmediata en un $33 \%$. Se verificó aumento de capacidad vesical postoperatoria de $72 \%$ y $52 \%$ para el séptimo y decimoquinto día, respectivamente. Estos porcentajes fueron estadísticamente significativos con 95\% de confianza. Se presentaron 2 lesiones vesicales como complicación transoperatoria y un absceso como complicación postoperatoria.

Conclusiones: El modelo animal en conejos para la técnica de autoaumento vesical por laparoscopia es un modelo factible para enseñanza, entrenamiento y aplicación.

Palabras clave: Laparoscopia. Autoaumento vesical. Modelo experimental.

\section{ABSTRACT}

LAPAROSCOPIC VESICAL AUTOAUGMENTATION: AN ANIMAL MODEL IN RABBITS (ORYCTOLAGUS CUNICULUS)

Introduction: Small contracted bladder remains a challenge in clinical urological practice and treatment options are not always effective. Urothelium sparing surgical techniques represent a reasonable therapeutical option. We aimed to evaluate the feasibility of a rabbit model (Oryctolagus cuniculus) in the teaching and training setting for laparoscopic vesical autoaugmentation.

Methods: Transperitoneal laparoscopic bladder autoaugmentation was performed in five New Zealand male rabbits (Oryctolagus cuniculus). A three port technique was used for all cases. Circumferential detrusorectomy was performed with vascular pedicles sparing, subsequently submucosal detailed blunt dissection allowed the creation of a bladder diverticulum which was verified intraoperatively. Eye-ball cystometry was performed preoperatively and postoperatively to verify bladder capacity variations. Postoperatively cystometry was done immediately after the procedure and 7 and 15 days postoperatively. Statistical analysis was performed with T-student model. A p-value $<0.05$ was considered of significance for the analysis. Surgical procedure and perioperative animal care was minutely precised according to the Guidelines of the Institute of Laboratory Animal Resources, Commission on Life Sciences, National Research Council.

Results: Five New Zealand male rabbits (Oryctolagus cuniculus) were surgically treated as described. Median operative time was 68.8 minutes (range 44 -120). Median preoperatory bladder capacity for the series was 25.8 cc (7-52). Median postoperative bladder capacity was diminished in 33\% immediately after the operation. Then, bladder capacity augmented $72 \%$ and $52 \%$ for $7^{\text {th }}$ and $15^{\text {th }}$ postoperative days, respectively. Percentage variation in bladder capacity is statistically significant. Two operative complications (mucosal tear) were reported. A postoperatory abscess was observed in one animal.

Conclusion: The rabbit (Oryctolagus cuniculus) model for the laparoscopic bladder autoaugmentation is feasible for teaching, training and research. Bigger series and longer follow-up should be addressed.

Keywords: Laparoscopy. Bladder autoaugmentation. Experimental model. 
$\mathrm{D}$ entro del campo de la cirugía reconstructiva vesical se encuentra la realización de procedimientos de aumento vesical con diferentes indicaciones así como variabilidad de técnicas. Una de éstas, es el autoaumento, indicada en vejigas de pobre distensibilidad e hiperrefléxicas con disminución de capacidad.

La vejiga contraída o con poca capacidad puede observarse en déficit neurológicos (mielomeningocele, lesiones traumáticas de médula espinal y enfermedades desmielinizantes). Existen condiciones patológicas no neurogénicas que pueden producir una vejiga contraída, como cistitis intersticial y cistitis actínica ${ }^{1}$.

$\mathrm{El}$ aumento con métodos quirúrgicos mediante el uso de segmentos gastrointestinales ha sido usado en el manejo de la compleja disfunción del tracto urinario inferior.

El incremento de la capacidad vesical mediante el uso de tejido urotelial nativo constituye la técnica de autoaumento. La creación de un gran divertículo vesical mediante la disección del detrusor en el domo de la vejiga, permite aumentar la capacidad de almacenamiento y reducir la presión intravesical ${ }^{1}$.

El primer reporte de caso de autoaumento vesical descrito en la literatura fue realizado por Mahony y Laferte. La primera serie de autoaumento vesical fue publicada en 1989 por Cartwright y Snow en pacientes pediátricos ${ }^{3}$; estos autores diseñaron su técnica en un modelo animal canino. Posteriormente Stothers et al., describieron el autoaumento vesical mediante la incisión del detrusor sin realizar escisión de las fibras musculares 1. Johnson et al., demostraron en el modelo animal del conejo que incidir o realizar exéresis del detrusor tenía un efecto beneficioso desde el punto de vista urodinámico ${ }^{1}$.

Ehrlich y Gershman describieron por primera vez, el abordaje transperitoneal laparoscópico en un paciente pediátrico con capacidad vesical limitada y elevada presión, asociada a incontinencia ${ }^{3}$.

La cirugía mínimamente invasiva impone tareas específicas que requieren habilidades psicomotoras que tienen que ver con el ambiente quirúrgico videoendoscópico. Los métodos tradicionales de enseñanza de la cirugía a cielo abierto no permiten la adquisición de las habilidades necesarias para la cirugía mínimamente invasiva. Cuando se realizan cirugías endoscópicas, los individuos en entrenamiento no pueden imitar las maniobras quirúrgicas de sus entrenadores sin antes haber manipulado los instrumentos laparoscópicos y haberse expuesto al ambiente quirúrgico endoscópico bidimensional que es inicialmente desorientador ${ }^{3,4}$.

Los modelos animales permiten al cirujano crear el neumoperitoneo y trabajar en la cavidad abdominal a un costo razonable, simulando diferentes escenarios $^{3,4}$.

\section{MATERIAL Y MÉTODOS}

El presente estudio está basado en una investigación de carácter experimental, con basamento en un estudio de corte descriptivo, en el cual se utilizaron los siguientes análisis: El descriptivo, en el cual se calcularon las medidas de tendencia central y dispersión; y el de significación o validación estadística inferencial mediante el cual se comparan proporciones con base en la distribución normal para variables discretas; para variables continuas se utilizó la comparación por diferencia de medias con varianzas desiguales basadas en la distribución T-student. Todos los contrastes de hipótesis se realizaron con un $\alpha=0,05$, es decir, una confianza del 95\%. El cálculo del p-valor para todos los contrastes seguirá la siguiente regla de validación: el contraste será significativo si el pvalor es menor de 0,05.

La intervención quirúrgica y el cuidado postoperatorio de los animales se realizó de acuerdo a los parámetros establecidos por la Guía para el Cuidado y Uso de los Animales de Laboratorio, editada por el Consejo Nacional de Investigación de Estados Unidos ${ }^{3}$ y mantenidos en jaulas individuales según criterios del Instituto de Cirugía Experimental, Facultad de Medicina de la Universidad Central de Venezuela, en condiciones estándar de laboratorio (temperatura $20^{\circ} \mathrm{C}$, humedad relativa de $70 \%$ y una relación día/noche de 12/12 horas).

Se utilizaron cinco conejos machos, enteros, raza Nueva Zelanda (Oryctolagus cuniculus), desparasitados, provenientes del bioterio del Insituto Nacional de Higiene, con un peso entre 1.900grs. y 3.100grs, alimentados según criterios del Instituto de Cirugía Experimental.

\section{Procedimiento Anestésico}

Se realizó un ayuno preoperatorio de doce (12) horas para el alimento y dos (2) para ingesta de agua. Luego de la premedicación con Clorhidrato de 
Xilacina $(5 \mathrm{mg} / \mathrm{Kg} \mathrm{IM})^{1-3}$, se indujo la anestesia con Clorhidrato de Ketamina $\left(44 \mathrm{mg} / \mathrm{Kg}\right.$ IM) ${ }^{11-13}$ procediendo al mantenimiento con una máscara adaptada a un sistema Mapleson E modificado (circuito de Jackson-Rees) con una bolsa de reserva de 5lts, utilizando una máquina de anestesia inhalatoria de circuito semiabierto con un vaporizador de cálculo directo (Fluotec Mark III) 14 con halotano (0,8-1,2\%) y una mezcla de oxígeno/óxido nitroso en una relación 33:66, con ventilación espontánea ${ }^{11-13}$.

A continuación se procede a realizar la cistometría Simple ${ }^{15}$ con llenado pasivo a gravedad, 50 microgotas por minuto, de solución fisiológica con reservorio de solución colocado a $40 \mathrm{~cm}$ sobre la pelvis del animal hasta observar fuga de líquido alrededor de la sonda, con la consecuente medición del volumen instilado para ese momento; el cual correspondería a la capacidad vesical máxima.

El procedimiento operatorio se realiza mediante la creación de neumoperitoneo $(5-8 \mathrm{mmHg})$ por técnica de Hasson con portal de $10 \mathrm{~mm}$, supraumbilical, línea media del abdomen. A continuación se precede a la colocación de dos (2) puertos accesorios de $5 \mathrm{~mm}$ bajo visión directa, en ambos flancos. Se revisa la cavidad abdominal y localiza la vejiga y se realiza disección roma a través del músculo detrusor para la creación de divertículo vesical e incisión del músculo detrusor $(3 \mathrm{~cm})$ en la línea media vesical en sentido transversal sobre los vasos de irrigación vesical, a continuación se crean los colgajos bilaterales (anterior y posterior) y se procede a su resección y extracción. Finalmente, se verifica la indemnidad de la mucosa vesical expuesta y se procede terminar el procedimiento y realizar cistometría simple postoperatoria.

Las complicaciones intraoperatorias de perforación de mucosa vesical se resolvieron laparoscópicamente con sutura intracorpórea, dejándose sonda de Foley 10 Fr por 48 horas y collar "isabelino" para prevenir el retiro de la misma.

Seguimiento postoperatorio

Se delimitó el seguimiento postoperatorio a quince días, durante los cuales se realizaron mediciones de la capacidad vesical, en dos períodos: a los 7 y 15 días con la finalidad de evaluar modificaciones.

\section{RESULTADOS}

Cinco conejos machos, raza New Zealand, peso promedio $2.640 \mathrm{~g}$ (1.900-3.100), fueron operados por técnica laparoscópica transperitoneal de tres puertos con neumoperitoneo de $5-8 \mathrm{mmHg}$, en un tiempo promedio de 68,8 minutos (rango 44-120) (Tabla 1).

Se realizó la medición de la capacidad vesical preoperatoria en cada animal, por técnica de cistometría simple, obteniéndose un promedio de $25,8 \mathrm{cc}$ (7-52). A continuación, se realizó el procedimiento quirúrgico. Una vez realizado el autoaumento y formado el divertículo vesical, se evaluó la retracción de los colgajos musculares y se verificó la indemnidad de la mucosa. Los colgajos fueron dejados sin fijación (Fig. 1).

Durante el acto quirúrgico se presentó un sangrado estimado promedio de $0,86 \mathrm{cc}(0,5-1)$ y se pre-

Tabla 1. Registro de datos

\begin{tabular}{|c|c|c|c|c|c|}
\hline Unidad de observación & 1 & 2 & 3 & 4 & 5 \\
\hline Sexo & M & M & M & M & M \\
\hline Peso (g) & $1900 / 1770 / 1700$ & $2200 / 2000 / 2200$ & $3000 / 3000 / 2800$ & $3000 / 3000 / 2900$ & $3100 / 3200 / 3100$ \\
\hline Tiempo $\mathrm{Qx}$ & 65 & $120^{\prime}$ & $44^{\prime}$ & 55 & 60 \\
\hline Sangrado estimado & $1 \mathrm{cc}$ & $1 \mathrm{cc}$ & $0,5 \mathrm{cc}$ & $0,8 \mathrm{cc}$ & $1 \mathrm{cc}$ \\
\hline Complicaciones intraoperatorias & No & Sí & No & No & Sí \\
\hline Capacidad vesical preoperatoria & $7 \mathrm{cc}$ & $32 \mathrm{cc}$ & $52 \mathrm{cc}$ & $10 \mathrm{cc}$ & $28 \mathrm{cc}$ \\
\hline Capacidad vesical postorperatoria inmediata & $8,5 \mathrm{cc}$ & - & $32 \mathrm{cc}$ & $11 \mathrm{cc}$ & - \\
\hline Diferencia capacidad vesical & $+1,5 \mathrm{cc}$ & - & $-20 \mathrm{cc}$ & $+1 \mathrm{cc}$ & - \\
\hline Neumoperitoneo & $5-8 \mathrm{mmHg}$ & $5-8 \mathrm{mmHg}$ & $5-8 \mathrm{mmHg}$ & 5-8 mmHg & $5-8 \mathrm{mmHg}$ \\
\hline Evolución & Satisfactoria & Satisfactoria & Satisfactoria & Satisfactoria & Satisfactoria \\
\hline Observaciones & $\begin{array}{l}\text { Absceso en trócar de } \\
10 \mathrm{~mm} \text { a los } 7 \text { días }\end{array}$ & - & - & - & - \\
\hline Capacidad vesical a los 7 días & $22 \mathrm{cc}$ & $53 \mathrm{cc}$ & $32 \mathrm{cc}$ & $57 \mathrm{cc}$ & $58 \mathrm{cc}$ \\
\hline Capacidad vesical a los 15 dias & $29 \mathrm{cc}$ & $26 \mathrm{cc}$ & $20 \mathrm{cc}$ & $61 \mathrm{cc}$ & $61 \mathrm{cc}$ \\
\hline
\end{tabular}




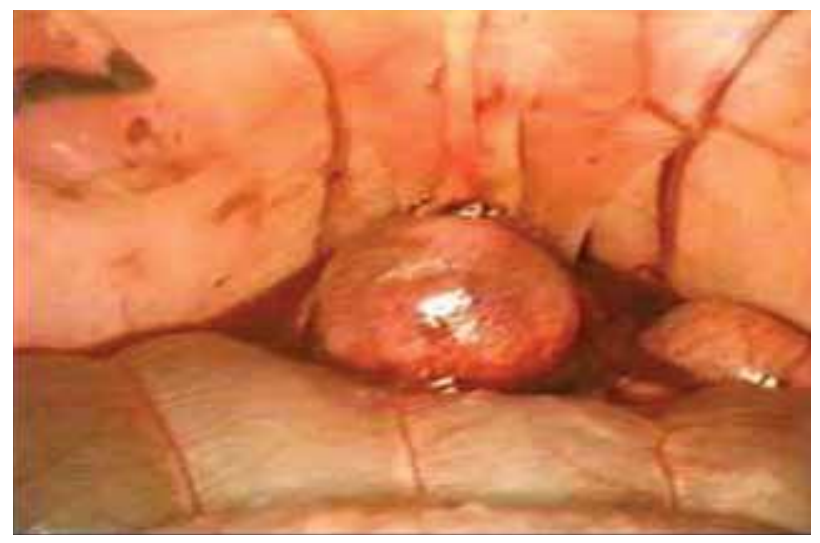

FIGURA 1. Diverticulo vesical creado.

sentaron dos lesiones intraoperatorias de mucosa vesical que fueron reparadas de manera inmediata con sutura laparoscópica intracorpórea.

Una vez completado el procedimiento, se realizó la medición de la capacidad vesical postoperatoria inmediata con un promedio de 17,17 cc $(8,5-32)$ para tres animales. En los dos animales restantes, la cistometría simple inmediata no fue realizada ante el evento de lesión vesical intraoperatoria, dejándose sonda vesical por espacio de 48 horas. (Tabla 1) En la evolución postoperatoria, se observó un absceso en el portal 1 del animal $N^{\circ} 1$. Los animales restantes evolucionaron sin complicaciones.

La capacidad vesical fue medida por cistometría simple de manera mediata y tardía, a los 7 y 15 días, respectivamente. Para la medición al séptimo día, la capacidad vesical promedio fue de 44,4 cc (22-58) y para el día quince la capacidad vesical promedio fue de 39,4 cc (20-61) (Fig. 2).

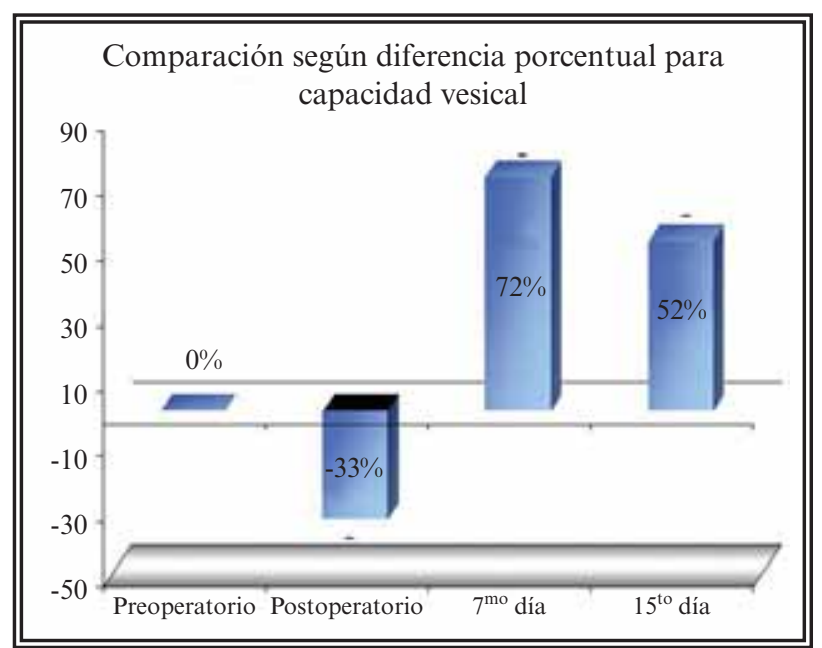

FIGURA 2. Diferencia porcentual de capacidad vesical.

\section{DISCUSIÓN}

La vejiga pequeña y contraída representa un cuadro clínico de difícil manejo urológico. La cirugía preservadora de urotelio se presenta como una opción terapéutica que, evitando las complicaciones descritas con el uso de segmentos gastrointestinales, permite de manera efectiva el aumento de la capacidad vesical. El autoaumento de vejiga fue descrito originalmente por Cartwright y Snow en un modelo animal canino, donde se realizó la escisión parcial del detrusor, lográndose aumento de capacidad vesical y bajas presiones ${ }^{2}$. Estos mismos autores presentan reportes posteriores de la aplicación clínica de su modelo experimental con resultados alentadores ${ }^{16}$. Mahony y Laferte habían propuesto la creación de múltiples miotomías a nivel del detrusor pero no se difundió su técnica. Eliçevik et al. plantearon el uso del conejo como modelo animal en un cuadro comparativo de diferentes técnicas quirúrgicas aplicadas para el aumento y plastia de la vejiga, donde se verifican resultados que validan este modelo animal para la cirugía a cielo abierto.

Ehrlich y Gershman reportaron la realización de autoaumento vesical por técnica laparoscópica en un paciente pediátrico con vejiga neurogénica, caracterizado por su baja capacidad y alta presión intravesical asociada con incontinencia ${ }^{5}$. De la misma forma, McDougall et al. reportaron un caso de vejiga neurogénica post-traumática en una paciente de 26 años, caracterizada por altas presiones y baja capacidad tratada con autoaumento realizado por vía laparoscópica, llegando a obtenerse una capacidad total de $350 \mathrm{cc}^{1}$

La presente serie muestra un proyecto experimental en conejos para el desarrollo de técnicas de autoaumento vesical realizada mediante cirugía mínimamente invasiva. Se escogió el conejo macho como unidad de observación por poseer características anatómicas ideales que facilitan el procedimiento de sondeo vesical y requerimientos de mantenimiento poco complejos, permitiendo así que las técnicas descritas sean reproducibles para su aplicación en el desarrollo de procedimientos de cirugía reconstructiva vesical, como ha sido demostrado por otros autores ${ }^{4}$.

En nuestra serie, la capacidad vesical disminuyó en un 33\% para las tres unidades de observación en las que se realizó la medición en el postoperatorio inmediato. Para el $7^{\circ}$ día se verificó un aumento de 
capacidad vesical en $72 \%$ de las unidades de observación y para el $15^{\text {to }}$, el aumento fue de un $52 \%$. Estos porcentajes de aumento vesical fueron estadísticamente significativos con 95\% de confianza (Fig. 2). Debemos reconocer que podría ser difícil obtener conclusiones objetivas a partir de 3 casos estudiados, si tomamos en cuenta las unidades de estudio en las que se realizó la evaluación post-operatoria.

La literatura describe la realización de evaluaciones urodinámicas y cistogramas en la validación de modelos experimentales animales para la cirugía de aumento vesical. Consideramos, que si bien los resultados obtenidos a través de estas pruebas son objetivos, la infraestructura necesaria para su realización aleja la posibilidad de reproducir éstos en todos los ambientes. Un procedimiento sencillo como la cistometría, permitió validar nuestra técnica quirúrgica sin afectar los costos.

Usualmente se describe la realización de la detrusorectomía en sentido longitudinal, desde el domo hasta el cuello vesical. En nuestra serie, se realizó el procedimiento en sentido transversal tomando en cuenta el patrón de irrigación de la vejiga del conejo. Esto permitió trabajar en un plano avascular y al mismo tiempo lograr un colgajo muscular bien irrigado que bien podría limitar el proceso de fibrosis postoperatorio.

\section{CONCLUSIÓN}

El modelo animal en conejos para la técnica de autoaumento vesical por laparoscopia es un modelo factible para enseñanza, entrenamiento y aplicación en nuestro país, por sus características anatómicas, de mantenimiento y su bajo costo permitiendo al cirujano desarrollar habilidades y destrezas in vivo, de manera segura y efectiva.

\section{REFERENCIAS}

1. McDougall E, Clayman R, Figenshau R, Pearle M. Laparoscopic retropubic auto-augmentation of the bladder. J Urol. 1995;153(1):123-126.

2. Cartwright PC, Snow BW. Bladder autoaugmentation: Partial detrusor excision to augment the bladder without use of bowel. J Urol. 1989;142(4): 1050-1053

3. Stothers L, Johnson H, Arnold W, Coleman G, Tearle H. Bladder autoaugmentation by vesicomyotomy in the pediatric neurogenic bladder. Urology. 1994;44(1):110-113.

4. Johnson HW, Nigro MK, Stothers ML. Laboratory variables of bladder autoaugmentation in an animal model. Urology. 1994;44(2):260-263.

5. Ehrlich RM, Gershman A. Laparoscopic seromyotomy (auto-augmentation) for non neurogenic bladder in a child: initial case report. Urology. 1993;42(2):175-178.

6. Melvin SW, Jonson JA, Ellison CE. Laparoscopic skills enhancement. Am J Surg. 1996;172(4):377-379.

7. Schiijven M, Jakimowicz J. Virtual reality surgical laparoscopic simulators. Surg Endosc. 2003;17(12):1943-1950.

8. Wolfe BM, Szabo Z, Moran ME, Chan P, Hunter JG. Training for minimally invasive surgery. Need for surgical skills. Surg Endosc. 1993;7(2):93-95.

9. Villegas L, Schneider BE, Callery MP, Jones DB. Laparoscopic skills training. Surg Endosc. 2003;17(12):1879-1888.

10. Guía para el cuidado y uso de los animales de laboratorio. Edited by the Institute of Laboratory Animal Resources, Commission on Life Sciences, National Research Council. Translated into Spanish by the Academia Nacional de Medicina; Mexico, 1999

11. Molinas C, Binda M, Mailova K, Koninckx P. The rabbit nephrectomy model for training in laparoscopic surgery. Hum Reprod. 2004;19(1): 185-190.

12. Holzinger F, Krähenbühl L, Schteingart C, Ton-Nu HT, Hofmann A. Use of a fluorescent bile acid to enhance visualization of the biliary tract and bile leaks during laparoscopic surgery in rabbits. Surg Endosc. 2001;15(2):209-212.

13. De Boer J, Archibald J, Downie H: Manual de Cirugía Experimental. Editorial El Manual Moderno, S.A., Mexico p. 67-68; 1979.

14. Warren R: Anestesia de animales domésticos. Primera edición. p328. Editorial Labor S.A., España. 1986.

15. Rayome RG. Simple urodynamic techniques. J Wound Ostomy Continence Nurs. 1995;22(1):17-26.

16. Cartwright PC, Snow BW: Bladder autoaugmentation: early clinical experience. J Urol. 1989;142(2 Pt 2):505-8; discussion 520-521.

Correspondencia autor: Dr. R.E. Sánchez-Salas Servicio de Urología. Hospital Universitario de Caracas.

Universidad Central de Venezuela. Caracas, Venezuela.

Tel.: 00582122658482

E-mai autor: raersas@hotmail.com

Información artículo: Original - Laparoscopia

Trabajo recibido: abril 2007

Trabajo aceptado: junio 2007 